\title{
Etiology of Influenza
}

\author{
Jian Wang
}

Human influenza virus is an enveloped single stranded RNA virus, belonging to the family of Orthomyxoviridae, and can be typed into type A, B, and C. The virus is spherical or filamentous in shape, with a diameter of 80-120 nm. The 3 types of human influenza virus show similar biochemical and biological properties. The viral envelope is composed of three layers: the inner layer of virus nucleocapsid containing nucleocapsid protein (NP), $\mathrm{P}$ protein and RNA; the middle layer of virus cyst envelope, composed of a lipoid layer and a layer of membrane protein (MP); and the outer layer of radiating spikes, composed of two different glycoproteins, hemagglutinin ( $\mathrm{H}$ or $\mathrm{HA}$ ) and neuraminidase ( $\mathrm{N}$ or $\mathrm{NA}$ ). Concerning the inner layer, NP is a type-specific soluble antigen (S antigen) with stable antigenicity. P protein (P1, P2, P3) may be polymerase necessary in transcription and replication of RNA. As for the middle layer, MP is also type-specific with stable antigenicity. And as for the outer layer, both of $\mathrm{H}$ and $\mathrm{N}$ have antigenicity and subtype specificity. $\mathrm{H}$ plays a role in adhering the virus to the surface of sensitive cells, and it can cause agglutination of erythrocytes. $\mathrm{N}$ contributes to detaching the virus from the surface of sensitive cells after its replication and it can hydrolyze terminal $\mathrm{N}$ - acetylneuraminic acid and mucin of receptor specific glycoprotein on the cell surface. The virus genome, together with the NP on its surface, constitutes the ribonucleoprotein (RNP) complex.

Based on the antigenicity of NP, human influenza virus is typed into type A, B, and C. According to the different $\mathrm{H}$ and $\mathrm{P}$ antigens, the same type of human influenza virus is further categorized into several subtypes. The antigenic variation of influenza virus refers to the change of $\mathrm{H}$ and $\mathrm{N}$ antigenic structures, mainly $\mathrm{H}$ antigen. Within any subtype of influenza virus, small variations often occur (quantitative), which is known as antigenic drift. The emergence of new subtype (qualitative) due to large antigenic variation of $\mathrm{H}$ and/or $\mathrm{N}$ antigen is known as antigenic shift, and the new subtype, known as a variant virus strain, can cause pandemic prevalence of influenza. During the 20th century, 4 pandemics of influenza occurred, all of which were caused by type A influenza virus. An obvious alternation exist between an old and a following new subtype of influenza virus. After a new subtype of influenza virus emerges to prevail in a region, the previous old subtype of influenza virus can no longer be isolated in the region. In addition, each subtype of influenza virus produces some variants. Type A influenza virus can be further divided into $16 \mathrm{H}$ subtypes (H1-16) and $9 \mathrm{~N}$ subtypes (N1-9). Human influenza is mainly related to subtypes of $\mathrm{H} 1, \mathrm{H} 2, \mathrm{H} 3$ as well as N1 and N2. Large and small variations also occur in type B influenza virus, but its subtypes have not been definitively divided. And antigenic variation of type $\mathrm{C}$ influenza virus has not been reported yet.

Influenza virus is intolerant to heat and can be inactivated at a temperature of $100{ }^{\circ} \mathrm{C}$ for $1 \mathrm{~min}$ or at a temperature of $56{ }^{\circ} \mathrm{C}$ for $30 \mathrm{~min}$. It is sensitive to ultraviolet rays and commonly used disinfectants, such as $1 \%$ formaldehyde, peracetic acid, and disinfectants containing chlorine. Influenza virus is tolerant to low temperature and dryness, and it can survive in vacuum drying environment or at a temperature of under $-20^{\circ} \mathrm{C}$. Chicken embryo culture is commonly applied to isolate the virus.

Type A influenza viruses of animal and human share some common antigenic components, but with no cross infection. Generally it can only infect human after antigenic conversion by infecting an intermediate animal host. However, it has been proved recently that some types of avian influenza virus can directly infect human via antigenic variation.
J. Wang

Affiliated First Hospital, Xinjiang Medical University,

Urumuqi, Xinjiang, China

e-mail: jeanw1265@163.com 